\title{
Pool Segmentation for Predicting Water Traps
}

\author{
Yusuke Yasui ${ }^{\text {a }}$ Sara McMains ${ }^{\text {a }}$ \\ ${ }^{a}$ University of California, Berkeley, USA \\ Thomas Glau ${ }^{\text {b }}$ \\ ${ }^{\mathrm{b}}$ Daimler AG Research \& Development, Stuttgart, Germany
}

\begin{abstract}
We propose a new method to detect water trap regions in voids of oriented polygonal models that approximate the geometry of mechanical parts. Since water traps decrease cleaning and draining efficiency, accurately predicting such regions allows re-orienting parts to reduce manufacturing time and cost. We construct a directed graph that captures the flow of water in voids of a 3D input model, based on a fast orientation-dependent volume segmentation approach. We can quickly find the water trap regions by analyzing the directed graph. Since we take a purely geometric approach to solve this problem without employing any physical simulation, even if the geometry of the voids is complicated, we can calculate water traps quickly.
\end{abstract}

Key words: Waterjet cleaning, Reeb graph, water traps, cleanability, slicing, segmentation, computational geometry

\section{INTRODUCTION}

As the complexity and precision of mechanical parts and assemblies have increased, the possibility of in-service failures caused by manufacturing-related hard particle contamination (such as detached burrs and chips from machining) has increased considerably. Reliably removing solid particle contaminants from the surfaces of mechanical parts has become increasingly important in the automotive industry. However, miniaturization and increased geometric complexity has made it more difficult to access all the surfaces of parts to remove contaminants.

In this paper, we consider a manufacturing planning problem that arises when cleaning with high-pressure water jets. Water jets are effective for removing contaminants from the surface of mechanical parts, but the water may become trapped inside the part if the geometry of voids is complex. Since contaminants may accumulate in such regions and trapped water must be drained after the cleaning, finding an orientation that minimizes the potential water trap regions is important to increase the cleaning efficiency and reduce the draining time and effort after cleaning.

We propose a new method to pre-identify the regions of cleaning-incompatible water traps in voids of mechanical parts using a geometric volume segmentation method, given the part orientation. We assume that the part geom- etry is given as a 2-manifold triangulated polygonal mesh and that the draining force applied to the water is only the gravitational force.

\section{PREVIOUS WORK}

To increase the efficiency of cleaning processes, analytical tools that predict cleaning effectiveness at the design and process planning stages are needed. Initial research has focused on understanding the effect of key cleaning process parameters $[3,2]$.

Since simulating multiphase fluid flow using computational fluid dynamics (CFD) can take hours to converge, some purely geometric approaches have been proposed for manufacturing planning with fluids. Bose and Toussaint proposed an algorithm to find an orientation for a gravity casting mold that minimizes the number of venting holes that need to be added to allow air to escape to insure a complete fill [4]. Aloupis et al. solved a 2D rotational draining problem for a closed polygon and a trapped single particle inside of the polygon, proposing an algorithm to find how many holes must be punctured to "drain" the particle [1]. For industrial applications of cleaning, on the other hand, we typically do not have the option of modifiying the part by adding venting or draining holes to eliminate air or water traps. Yasui and McMains proposed an algorithm to test 


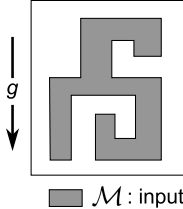

(a)

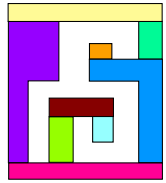

(e)

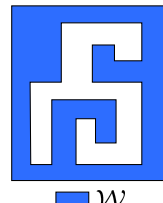

(b)

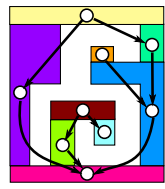

(f)

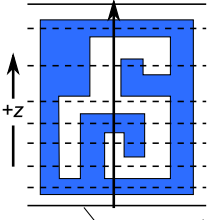

(c)

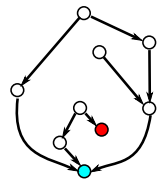

(g)

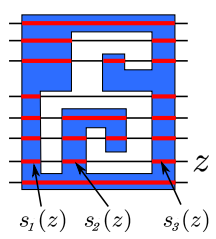

(d)

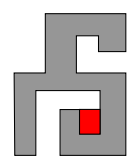

(h)
Fig. 1. Overview: (a) From input geometry $\mathcal{M}$, (b) we define the space $\mathcal{W}=\mathcal{B} \backslash \mathcal{M}$ where water could flow. (c) Using a sweep plane $p_{\text {sweep }}(z),(\mathrm{d})$ we track the evolution of connected slice components $s_{i}(z) \in \mathcal{W} \cap p_{\text {sweep }}(z)$. (e) At locations where slice components split or merge, we segment $\mathcal{W}$ into pools, and (f) assign directed edges that capture the water flow between pairs of pools. (g) From the constructed graph, we locate potential water trap regions. (h) We map the region(s) to input $\mathcal{M}$.

whether a given rotation axis can fully drain a workpiece when the workpiece is rotated around the axis [10]. The method we propose in this paper is also purely geometric, avoiding the computationally costly simulations of CFD.

\section{ALGORITHM OVERVIEW}

Our algorithm predicts regions in voids of the geometry where, for a given orientation, the effectiveness of cleaning with water jets will be compromised due to water traps. We assume throughout this paper that the part geometry has been rotated to the desired test orientation, so that gravity always acts vertically (i.e. down the z-axis).

Figure 1 illustrates an overview of our algorithm. Letting $\mathcal{M}$ be the geometry of the input model and $\mathcal{B}$ be a slightly enlarged bounding box that encloses $\mathcal{M}$, the space $\mathcal{W}$ where water flows can be represented as $\mathcal{W}=\mathcal{B} \backslash \mathcal{M}$. We split the space $\mathcal{W}$ horizontally into multiple regions called pools based on topological changes of $\mathcal{W}$ with respect to the z-axis. Then, we build a directed graph whose nodes correspond to the pools and whose edges connect two nodes if water flowing out of the source node's corresponding pool could enter the destination node's corresponding pool. We determine water trap regions by analyzing the directed graph.

The directed graph we construct is mathematically equivalent to a Reeb graph of a 3-manifold with boundary with respect to the height function (z-value). Hence, we could construct the directed graph from $\mathcal{W}$ using a Reeb graph construction algorithm $[8,9]$ and segment $\mathcal{W}$ into pools based on the Reeb graph constructed. However, since those Reeb graph construction approaches require the extra burden of tetrahedralizing $\mathcal{W}$, we propose an alternative efficient approach of segmenting $\mathcal{W}$ into pools and constructing the corresponding directed graph simultaneously in our work.

\section{Preliminaries}

We introduce some notation that we will use to explain how we split $\mathcal{W}$ into pools and add the directed edges between nodes corresponding to pools. We consider a sweep plane $p_{\text {sweep }}(\mathrm{z}=z)$ perpendicular to the $\mathrm{z}$-axis (i.e. the gravity direction) intersecting it at $z$. Given a sweep plane $p_{\text {sweep }}(z)$, we define the slice at $z, S(z)$, as the intersection of $\mathcal{W}$ and $p_{\text {sweep }}(z): S(z)=\mathcal{W} \cap p_{\text {sweep }}(z)$. As shown in Figure $1(\mathrm{~d})$, slice $S(z)$ may consist of multiple disconnected slice components, which in $3 \mathrm{D}$ will be $2 \mathrm{D}$ polygons (possibly with holes). We call these slice polygons. We denote the different slice polygons constituting $S(z)$ as $s_{i}(z)$ $(1 \leq i \leq|S(z)|)$.

Then, we let $\operatorname{proj}\left(s_{i}(z)\right)$ be the projection of $s_{i}(z)$ to the plane perpendicular to the z-axis, and the $z$-value just below $z$ be $z^{-}=z-\epsilon$. We let the z-value just above $z$ be $z^{+}=$ $z+\epsilon, \epsilon$ a positive infinitesimal number. Given a slice polygon $s_{i}(z) \in S(z)$, we define overlapping slice polygon(s) just below $s_{i}(z), S_{\text {below }}\left(s_{i}(z)\right)$, as the set of slice polygons $s_{j}\left(z^{-}\right) \in S\left(z^{-}\right)$such that $\operatorname{proj}\left(s_{i}(z)\right) \cap \operatorname{proj}\left(s_{j}\left(z^{-}\right)\right) \neq \emptyset$. Similarly, we define overlapping slice polygon(s) just above $s_{i}(z), S_{\text {above }}\left(s_{i}(z)\right)$, as the set of slice polygons $s_{j}\left(z^{+}\right) \in$ $S\left(z^{+}\right)$such that $\operatorname{proj}\left(s_{i}(z)\right) \cap \operatorname{proj}\left(s_{j}\left(z^{+}\right)\right) \neq \emptyset$.

Based on the cardinality of $S_{\text {below }}\left(s_{i}(z)\right)$ and $S_{\text {above }}\left(s_{i}(z)\right)$, the slice polygons just below and above $s_{i}(z)$, we classify each slice polygon $s_{i}(z)$ as one of four types as follows. Given a slice polygon $s_{i}(z)$, if $\left|S_{\text {below }}\left(s_{i}(z)\right)\right|=0$, we call $s_{i}(z)$ a beginning slice polygon since a new slice polygon appears as the sweep plane moves from $p_{\text {sweep }}\left(z^{-}\right)$ to $p_{\text {sweep }}\left(z^{+}\right)$. On the other hand, if $\left|S_{\text {above }}\left(s_{i}(z)\right)\right|=0$, we call $s_{i}(z)$ an ending slice polygon, since an existing slice polygon disappears as the sweep plane moves from $p_{\text {sweep }}\left(z^{-}\right)$to $p_{\text {sweep }}\left(z^{+}\right)$. If $\left|S_{\text {below }}\left(s_{i}(z)\right)\right| \geq 2$ and $\left|S_{\text {above }}\left(s_{i}(z)\right)\right| \geq 1$, or if $\left|S_{\text {below }}\left(s_{i}(z)\right)\right| \geq 1$ and $\left|S_{\text {above }}\left(s_{i}(z)\right)\right| \geq 2$, we call $s_{i}(z)$ a merge/split slice polygon since multiple slice polygons merge into one slice polygon and/or one slice polygon splits into multiple slice polygons as the sweep plane moves from $p_{\text {sweep }}\left(z^{-}\right)$to $p_{\text {sweep }}\left(z^{+}\right)$. Finally, if $\left|S_{\text {below }}\left(s_{i}(z)\right)\right|=$

$\left|S_{\text {above }}\left(s_{i}(z)\right)\right|=1$, we call $s_{i}(z)$ a no-change slice polygon, since no topological change of slice polygon $s_{i}(z)$ occurs as the sweep plane moves from $p_{\text {sweep }}\left(z^{-}\right)$to $p_{\text {sweep }}\left(z^{+}\right)$.

\section{Pool Segmentation Overview}

We define a pool as the union of no-change slice polygons bounded by either a beginning or a merge/split slice polygon from below and either an ending or a merge/split slice polygon from above. Given a slice polygon $s_{i}(z)$, we let $\operatorname{pool}\left(s_{i}(z)\right)$ be the pool $s_{i}(z)$ defines.

We segment $\mathcal{W}$ into pools using a sweep plane algorithm, where we imagine moving $p_{\text {sweep }}(z)$ from $\mathrm{z}=-\infty$ to $\mathrm{z}=+\infty$. If $\mathcal{W} \cap p_{\text {sweep }}(z)$ yields a beginning slice polygon, we generate a new pool bounded from below by the beginning slice polygon. If $\mathcal{W} \cap p_{\text {sweep }}(z)$ yields a no- 
change slice polygon, the no-change slice polygon $s_{i}(z)$ defines the pool $\operatorname{pool}\left(s_{j}\left(z^{-}\right)\right)$where $s_{j}\left(z^{-}\right) \in S_{\text {below }}\left(s_{i}(z)\right)$. If $\mathcal{W} \cap p_{\text {sweep }}(z)$ yields an ending slice polygon, we complete the corresponding existing pool, bounding it from above with the ending slice polygon. Finally, if $\mathcal{W} \cap p_{\text {sweep }}(z)$ yields a merge/split slice polygon, we complete the corresponding existing pool(s) by bounding from above with the merge/split slice polygon, and generate new pool(s) by bounding from below with the same merge/split slice polygon. Then, for $1 \leq i \leq\left|S\left(z^{-}\right)\right|$and for $1 \leq j \leq$ $\left|S\left(z^{+}\right)\right|$, we compute $\left(\operatorname{proj} s_{i}\left(z^{-}\right)\right) \cap \operatorname{proj}\left(s_{j}\left(z^{+}\right)\right)$. If there are $p$ and $q$ such that $\operatorname{proj}\left(s_{p}\left(z^{-}\right)\right) \cap \operatorname{proj}\left(s_{q}\left(z^{+}\right)\right) \neq \emptyset$, and $\operatorname{pool}\left(s_{p}\left(z^{-}\right)\right) \neq \operatorname{pool}\left(s_{q}\left(z^{+}\right)\right)$, we add a directed edge from the node corresponding to $\operatorname{pool}\left(s_{q}\left(z^{+}\right)\right)$to the node corresponding to $\operatorname{pool}\left(s_{p}\left(z^{-}\right)\right)$in the directed graph (Figure 1 (f)).

\section{Predicting Water Trap Regions}

After completing the sweep from $\mathrm{z}=-\infty$ to $\mathrm{z}=+\infty$, the space $\mathcal{W}$ is segmented into pools that are connected to each other in the graph by edges oriented in the direction of gravity if they are bounded by the same merge/split slice polygon. Each pool represents a region that could potentially be a water trap region (except the bottom-most pool, which represents the exterior of $\mathcal{M})$. Water flowing in $\mathcal{W}$ under gravity will flow between pools according to the directed edges. Once such flowing water reaches the bottom-most pool, since by construction it is outside the input geometry, we consider the water to be drained. Thus, as shown in Figure $1(\mathrm{~g})$, given a pool, if there is no path such that we can reach the bottom-most pool from the corresponding node, the pool is a potential water trap region (whether or not this water trap is actually formed depends upon the inflow location). Since we can compute the volume of water each pool can hold, we can also quantitatively evaluate a given part orientation by summing the volumes of pools that are determined to be water trap regions.

\section{POOL SEGMENTATION}

In this section, we describe the details of our pool segmentation algorithm summarized above, given a 2-manifold triangulated input mesh $\mathcal{M}$.

From $\mathcal{M}$, we can easily obtain the corresponding $\mathcal{W}$ by flipping the orientation of the triangles in $\mathcal{M}$ and introducing six rectangles that represent the enlarged axis-aligned bounding box $\mathcal{B}$. Each rectangle should be split into two triangles such that all the faces of $\mathcal{W}$ are represented by triangles as well.

To implement the pool segmentation algorithm, we have to know for which values of $z$ beginning, ending, and merge/split slice polygons occur. We determine all of these values of $z$ by tracking the evolution of the boundary of slice polygons. Even when a slice polygon boundary appears, disappears, merges, or splits, the corresponding slice polygon does not necessarily appear, disappear, merge, or split (e.g. because the boundary could correspond to a hole in a polygon). However, when a slice polygon appears, disappears, merges, or splits, the corresponding slice polygon boundary does also appear, disappear, merge, or split. Therefore, checking all the values of $z$ where a slice polygon boundary appears, disappears, merges, or splits is sufficient to determine all the values of $z$ where beginning, ending, and merge/split slice polygons occur.

We track the evolution of slice polygon boundaries by modifying McMains' sweep plane slicing algorithm [6]. Observing that slice polygon boundaries appear, disappear, merge, or split only when the sweep plane passes through one of the vertices of the input polygonal mesh, they showed that all such changes can be identified as long as all vertices are checked in ascending order of z-coordinate (vertices whose $\mathrm{z}$-coordinates are the same can be processed in arbitrary order without affecting the final result). In other words, when $\mathcal{W} \cap p_{\text {sweep }}(z)$ yields any of beginning, ending, and/or merge/split slice polygons, $p_{\text {sweep }}(z)$ always intersects with one of the vertices in $\mathcal{W}$.

\section{Boundary Cycles}

Our modified sweep plane slicing algorithm tracks the evolution of slice polygon boundaries and determines when a slice polygon boundary appears, disappears, merges, or splits. For this purpose, we manage a status structure called the boundary cycle (Figure 2). Each boundary cycle consists of a set of triangles. Any triangle in $\mathcal{W}$ is visited three times during sweeping since each triangle has three vertices. Given a triangle, when it is visited for the first time, the triangle is inserted into a boundary cycle. When it is visited for the third time, the triangle is deleted from the boundary cycle. Since we process each vertex in ascending order of zcoordinate, triangles currently in a boundary cycle always intersect the current sweep plane.

Each slice polygon boundary at $z$ is represented by a closed polygonal chain on $p_{\text {sweep }}(z)$. Each line segment constituting the closed polygonal chain is defined by the intersection between $p_{\text {sweep }}(z)$ and a triangle. Letting $V(z)$ be the set of vertices in $\mathcal{W}$ whose z-coordinate is $z$, a set of triangles in a boundary cycle defines a slice polygon boundary at $z$ where $V(z)=\emptyset$ (i.e. where $p_{\text {sweep }}(z)$ does not intersect any vertices in $\mathcal{W}$ ) as shown in Figure 2. Therefore, the number of line segments constituting a slice polygon boundary is equal to the number of triangles in the corresponding boundary cycle. For such $z$, there is exactly one boundary cycle for each slice polygon boundary. For $z$ where $V(z) \neq \emptyset$ (i.e. where $p_{\text {sweep }}(z)$ intersects with at least one vertex in $\mathcal{W})$, a set of triangles in a boundary cycle does not necessarily define a slice polygon boundary since some triangles in the boundary cycle may be parallel to the sweep plane; the intersection between such a triangle and $p_{\text {sweep }}(z)$ is not a line segment. However, this limitation does not become a problem because, to determine the 

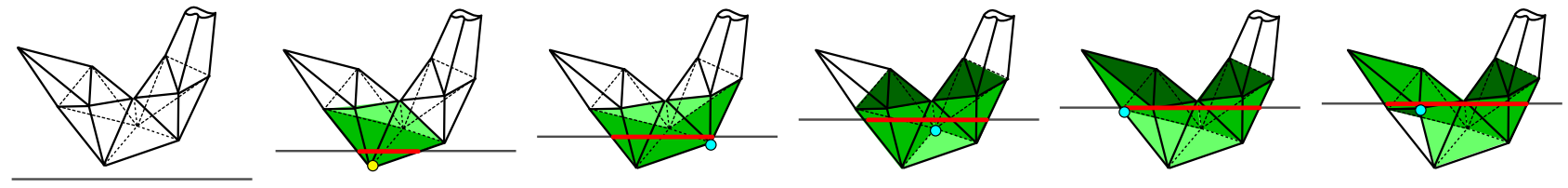

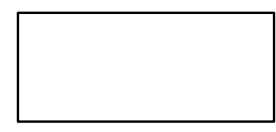

(a)
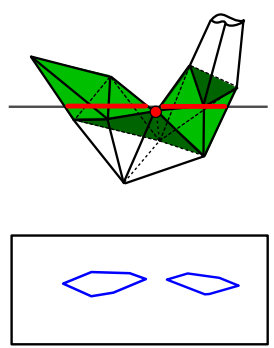

(g)

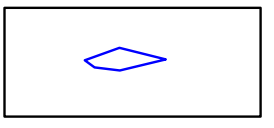

(b)
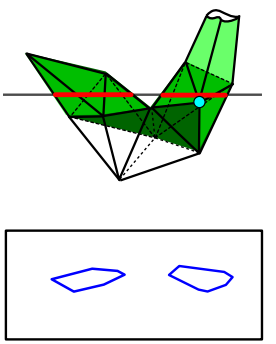

(h)

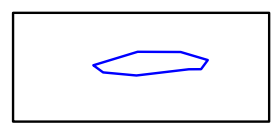

(c)
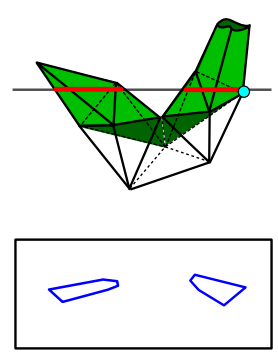

(i)

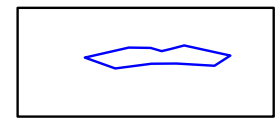

(d)
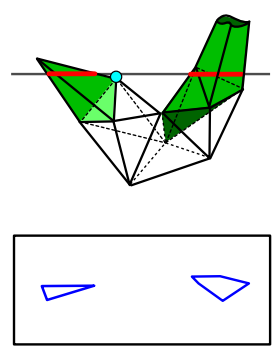

(j)

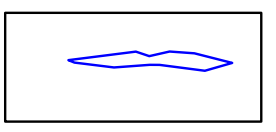

(e)
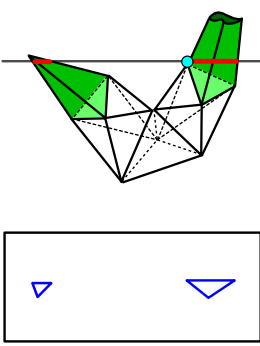

(k)

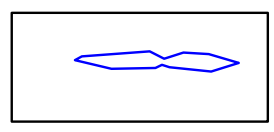

(f)
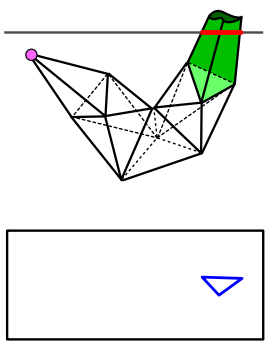

(I)

o beginning vertex $\circ$ ending vertex $\circ$ no-change vertex $\circ$ merge/split vertex

Fig. 2. Figures (a)-(l) illustrate how the triangles in boundary cycles are updated as the sweep plane moves from bottom to top over a portion of $\mathcal{W}$. The top drawing in each subfigure shows the set of triangles (colored) currently in boundary cycles just after the sweep plane processes the indicated vertex; the bottom drawing in each subfigure shows the corresponding slice polygon boundaries, with the sweep plane shown as a rectangle.

type of slice polygons at $z$ where $V(z) \neq \emptyset$, it is sufficient to consider the slice polygons just below and just above vertices in $V(z)$ (i.e. at $z^{-}$and $z^{+}$) as explained above. During sweeping from $\mathrm{z}=-\infty$ to $\mathrm{z}=+\infty$, we track the evolution of slice polygon boundaries by tracking the set of triangles in each boundary cycle.

\section{Boundary Cycle Management}

Boundary cycles are generated, completed, or updated when we process each vertex in $\mathcal{W}$. As McMains et al. showed [6], we can classify each vertex into one of four types: beginning vertex, ending vertex, no-change vertex, and merge/split vertex. A beginning vertex is where a new boundary cycle is generated. An ending vertex is where an existing boundary cycle is completed. A no-change vertex is where some triangles may be deleted from and inserted into an existing boundary cycle. A merge/split vertex is where multiple boundary cycles merge into one boundary cycle or one boundary cycle splits into multiple boundary cycles. At a merge/split vertex, we complete existing boundary cycle(s) and generate new boundary cycle(s) according to the merge or split. Appendix A and B describes, for a given $V(z)$, how to classify each vertex $v \in V(z)$ into one of the four types, and generate, complete, and update boundary cycles accordingly. When $\mathcal{W} \cap p_{\text {sweep }}(z)$ yields a beginning, an ending, or a merge/split slice polygon, $p_{\text {sweep }}(z)$ always intersects with a beginning, an ending, or a merge/split vertex, respectively (but not vice versa).

When a new boundary cycle is generated at a beginning vertex, a new slice polygon boundary appears (Figure 2 (b)); when an existing boundary cycle is completed at an ending vertex an existing slice polygon boundary disappears (Figure $2(1)$ ); and, when multiple boundary cycles merge into one boundary cycle or one boundary cycle splits into multiple boundary cycles at a merge/split vertex, multiple slice polygon boundaries merge into one slice polygon boundary or one slice polygon boundary splits into multiple slice polygon boundaries (Figure $2(\mathrm{~g})$ ).

We update triangles in an existing boundary cycle at a no-change vertex (Figure 2 (c)-(f) and (h)-(k)). We consider two boundary cycles at different values of $z$ to be the same boundary cycle if one is obtained from the other by processing only no-change vertices. Thus, a new boundary cycle will be generated at a beginning vertex or a merge/split vertex and an existing boundary cycle will be completed at an ending vertex or a merge/split vertex.

\section{Boundary Cycle Classification}

As shown in Figure 3, a slice polygon may be bounded by more than one slice polygon boundary. More specifically, a slice polygon is always bounded by one outer slice polygon boundary plus zero or more inner slice polygon boundaries. Given a slice polygon $s_{i}(z)$, we let $\partial s_{i}(z)$ be the set of slice polygon boundaries that bound $s_{i}(z)$. We also let $\left(\partial s_{i}(z)\right)_{1}$ be the outer slice polygon boundary and $\left(\partial s_{i}(z)\right)_{j}(j \geq$ 2 ) be the inner slice polygon boundaries of $s_{i}(z)$. Then, $\partial s_{i}(z)=\left\{\left(\partial s_{i}(z)\right)_{1}, \cdots,\left(\partial s_{i}(z)\right)_{\left|\partial s_{i}(z)\right|}\right\}$.

A boundary cycle is classified as either an outer boundary cycle or an inner boundary cycle depending on whether the intersection between triangles in the boundary cycle and 


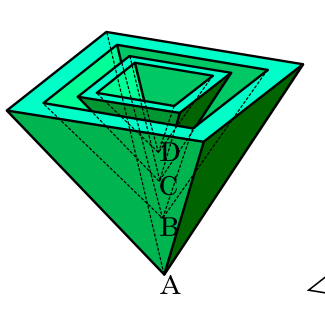

(a)

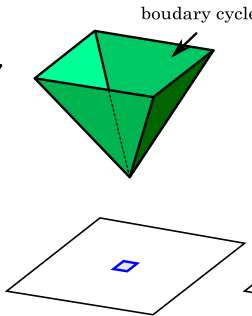

(b)

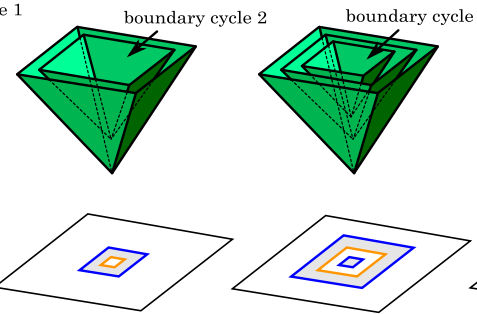

(c) (d)

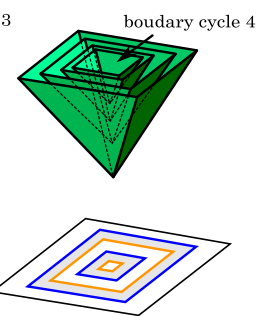

(e)

- outer slice polygon boundary

inner slice polygon boundary

Fig. 3. Each of subfigures (b)-(e) shows the boundary cycles and the corresponding slice polygon boundaries on the sweep plane just after processing vertices $A, B, C$, and, $D$ shown in (a), respectively. Boundary cycles 1 and 3 are outer boundary cycles; Boundary cycles 2 and 4 are inner boundary cycles. For each slice polygon, the inner boundary cycles that define the inner slice polygon boundaries are associated with the outer boundary cycle that defines the outer slice polygon boundary. Boundary cycle 2 is associated with boundary cycle 1 ; boundary cycle 4 is associated with boundary cycle 3 .

$p_{\text {sweep }}(z)$ define an outer or an inner slice polygon boundary. An inner boundary cycle is always associated with an outer boundary cycle that immediately encloses the inner boundary cycle (Figure 3).

For a given $z$, a boundary cycle is classified as an outer boundary cycle or an inner boundary cycle by shooting a ray perpendicular to the $\mathrm{z}$-axis from an arbitrary point at $z$ on a triangle in the boundary cycle and counting the number of intersections between the ray and triangles in $\mathcal{W}$, excluding triangles in the boundary cycle that we are testing. If it is even, the boundary cycle is an outer boundary cycle. If it is odd, it is an inner boundary cycle. For each inner boundary cycle, we can find the outer boundary cycle immediately enclosing the inner boundary cycle by counting the number of intersections with triangles in each outer boundary cycle. If there is an outer boundary cycle where the number of the intersections is odd, the outer boundary cycle encloses this inner boundary cycle. If multiple such enclosing boundary cycles exist, the one with the closest intersection is the immediately enclosing one.

\section{Pool Segmentation Implementation}

Now, we describe how to implement pool segmentation. Previously, we defined that a pool is the union of no-change slice polygons bounded by either a beginning or a merge/split slice polygon from below and either an ending or a merge/split slice polygon from above. We have observed that we can determine where these slice polygons occur during sweeping by tracking the evolution of boundary cycles. Therefore, we construct a pool according to generation, completion, and updating of boundary cycles. In practice, we construct a pool by finding its boundary. Specifically, the side of a pool is defined by triangles from $\mathcal{W}$, possibly trimmed. The bottom and top face of a pool is defined by the slice polygons where the pool is generated and completed.

Each pool is defined by one outer boundary cycle plus zero or more inner boundary cycles. Triangles in such boundary cycles form the sides of the pools. Each of these

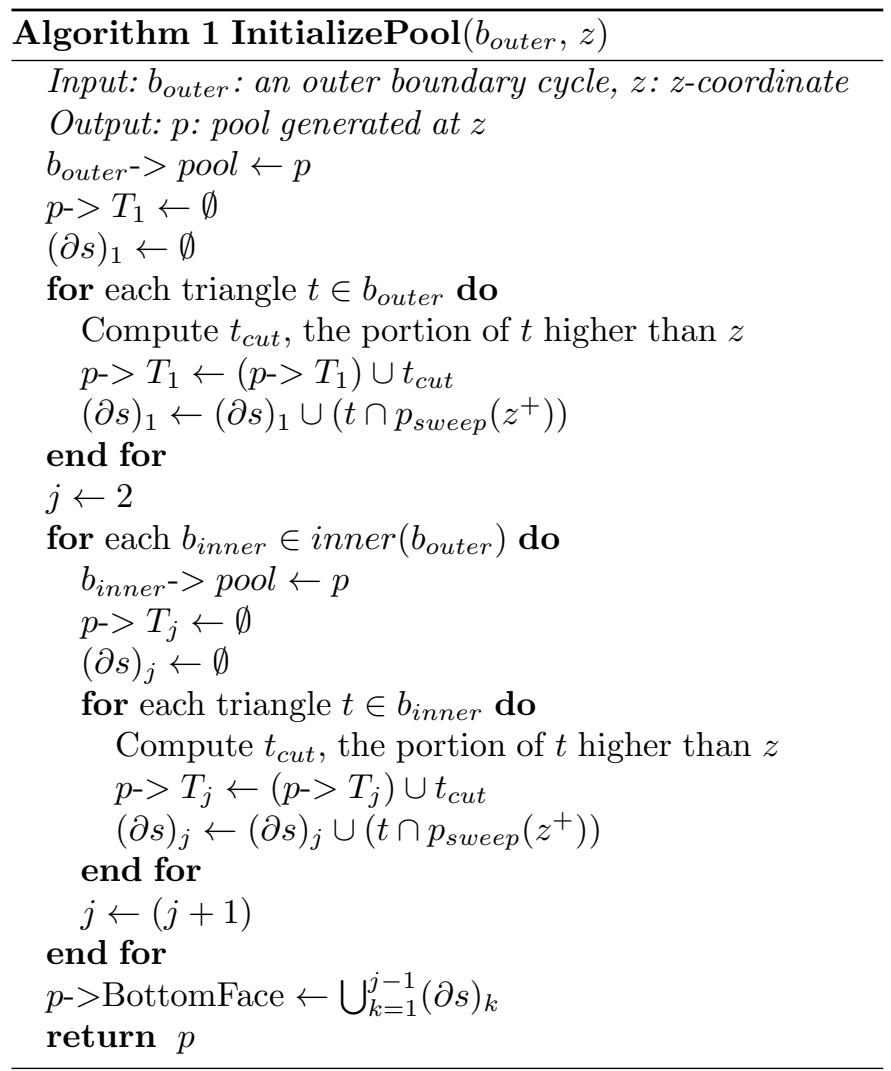

triangles is trimmed if a portion of the triangle is lower than the lower bound z-coordinate and/or higher than the upper bound $\mathrm{z}$-coordinate of the pool. Intersection between the triangles and the sweep plane at the lower/upper bound $\mathrm{z}$-coordinate define the bottom face and the top face of the pool, respectively.

We generate a new pool $p$ at a z-coordinate $z$ where the slice topology changes by performing the following operations that initialize the pool defined by the outer boundary cycle $b_{\text {outer }}$ at $z$. Letting inner $\left(b_{\text {outer }}\right)$ be the set of enclosed inner boundary cycles associated with $b_{\text {outer }}$, first, we assign the triangles in $b_{\text {outer }}$ and each $b_{\text {inner }} \in \operatorname{inner}\left(b_{\text {outer }}\right)$ to $p$. Then, for each triangle assigned to $p$ at its lowest $\mathrm{z}-$ value, if a portion of the triangle is lower than $z^{+}$, we trim 


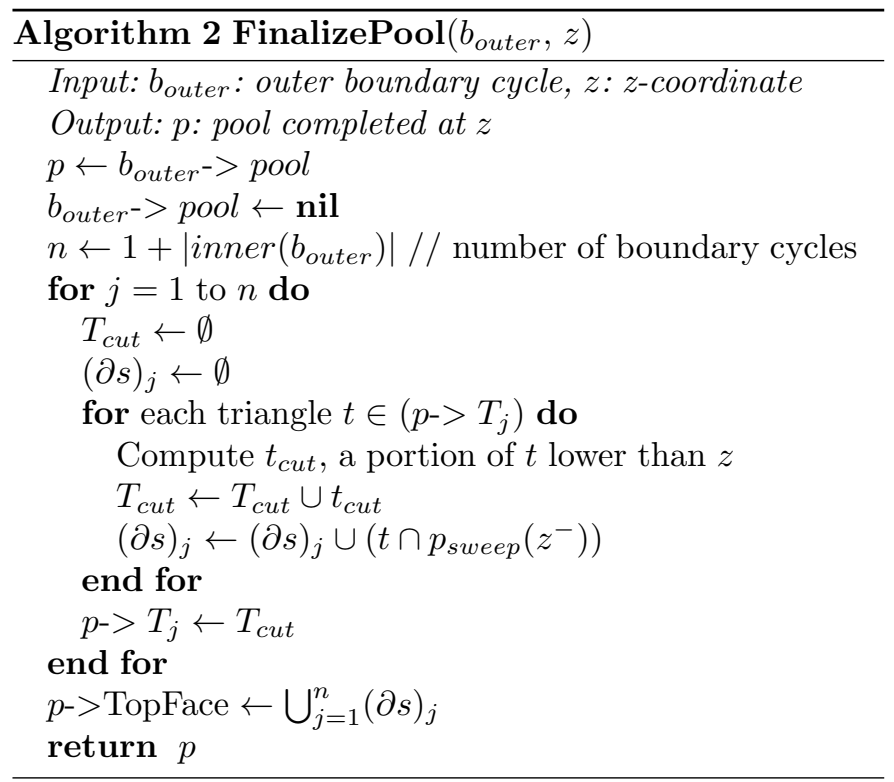

that portion (which may be the entire triangle). Then, we define the bottom face of $p$ by connecting the line segments defined by intersections between the trimmed triangles and $p_{\text {sweep }}\left(z^{+}\right)$. Note that, if $p$ is defined by $n$ boundary cycles, the bottom face consists of $n$ closed polygonal chains. The bottom face corresponds to a slice polygon $s_{i}\left(z^{+}\right)$and each of the closed polygonal chains corresponds to slice polygon boundary $\left(\partial s_{i}\left(z^{+}\right)\right)_{j}(1 \leq j \leq n)$, where $n=\left|\partial s_{i}\left(z^{+}\right)\right|$. Algorithm 1 gives the corresponding pseudocode for initializing a pool. In a similar manner, we complete an existing pool $p$ at a z-coordinate $z$ where the slice topology changes again by performing analogous operations to finalize the pool defined by the outer boundary cycle $b_{\text {outer }}$ at this $z$, the highest z-value for the pool. The difference is that we trim the triangles and define the top face at $z^{-}$, instead of the bottom face at $z^{+}$(Algorithm 2).

\section{Pool Segmentation, Simplified Case}

We describe how to construct each pool based on generation, completion, and updating of boundary cycles in detail. For the sake of simplicity of explanation, we assume that any slice polygon $s_{i}(z)$ is bounded by only one outer slice polygon boundary for a moment (i.e. $\left|\partial s_{i}(z)\right|=1$ for any $z$ and inner $\left(b_{\text {outer }}\right)=\emptyset$ for any outer boundary cycle $\left.b_{\text {outer }}\right)$. Thus, any boundary cycle we encounter during sweeping will always be an outer boundary cycle.

In this case, the appearance, disappearance, merging, and splitting of slice polygon boundaries always leads to appearance, disappearance, merging, and splitting of the corresponding slice polygons. Thus, for a given $z$ where $V(z) \neq \emptyset$, if a new boundary cycle is generated, we initialize a new pool defined by the boundary cycle. If an existing boundary cycle is completed, we finalize the existing pool defined by the boundary cycle.

For a given $z$ where $V(z) \neq \emptyset$, let $G, C$, and $U$ be the sets of new boundary cycles generated, completed, and updated at $v \in V(z)$, respectively. The specific algorithm to find $G$, $C$, and $U$ from $V(z)$ is described in Appendix C. A pool is constructed by the following rules.

For a given $z$ where $V(z) \neq \emptyset$ :

(i) For each updated boundary cycle $b \in U$, we add new triangles inserted into $b$ at $z$ to the pool defined by $b$.

(ii) For each completed boundary cycle $b \in C$, we finalize the pool defined by $b$.

(iii) For each newly-generated boundary cycle $b \in G$, we initialize the pool $p$ defined by $b$.

(iv) Let $P_{\text {init }}$ be the set of pools initialized and $P_{\text {final }}$ be the set of pools finalized at $z$. If $P_{\text {init }} \neq \emptyset$ and $P_{\text {final }} \neq \emptyset$, for $p_{i} \in P_{\text {init }}$ and for $p_{f} \in P_{\text {final }}$, we compare the bottom face of $p_{i}$ and the top face of $p_{f}$. If they overlap, we add a directed edge from the node corresponding to $p_{i}$ to the node corresponding to $p_{f}$ in the directed graph.

A series of steps to construct pools based on these rules is illustrated in Figure 4. When a new boundary cycle is generated, we initialize a new pool defined by the boundary cycle (Figure $4(\mathrm{~b})$ ). In this example, since the intersection between the triangles and the corresponding sweep plane becomes a point, no triangles are trimmed and the bottom face consists of a single point. When the triangles in the boundary cycles are updated, we assign the inserted triangles to the pool defined by their boundary cycles (Figure 4 (c)-(f) and (h)-(k)). When one boundary cycle splits into multiple boundary cycles (Figure 4) (g)), we finalize the pool defined by the existing boundary cycle (the purple pool). The portions of the triangles above the $\mathrm{z}$-coordinate of the indicated vertex are trimmed and the set of the corresponding intersection line segments defines the top face of the finalized pool. At the same time, we initialize new pools defined by the new boundary cycles (the yellow and blue pools). The triangles in the boundary cycles after processing the merge/split vertex indicated in the top of subfigure $(\mathrm{g})$ are assigned to the pools. The portions of the triangles below the z-coordinate of the indicated vertex are trimmed; the sets of corresponding intersection line segments define the bottom face of the newly generated pools. When a boundary cycle is completed, we finalize the pool defined by the boundary cycle (Figure 4 (l)). In this example, since the intersection between the triangles in the pool and the corresponding sweep plane becomes a point, no triangles are trimmed and the top face consists of a single point.

\section{Pool Segmentation, General Case}

We now remove the simplifying assumption that each slice polygon is bounded by only one outer slice polygon boundary. In the general case, a pool is defined by one outer boundary cycle and zero or more inner boundary cycles. Unlike in the previous simplified case, the appearance, disappearance, merging, or splitting of slice polygon boundaries does not necessarily lead to the appearance, disappearance, merging, and splitting of the corresponding slice polygons for the general case. For example, in Figure 3, the appearance of the inner slice polygon boundary does not lead to the appearance of a new slice polygon; the ap- 

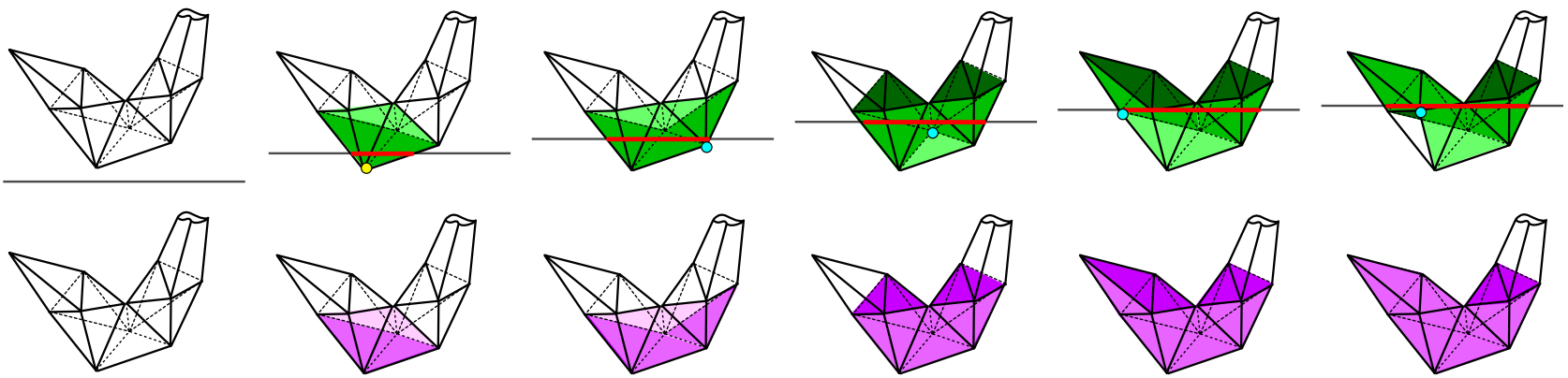

(a)

(b)

(c)

(d)

(e)

(f)
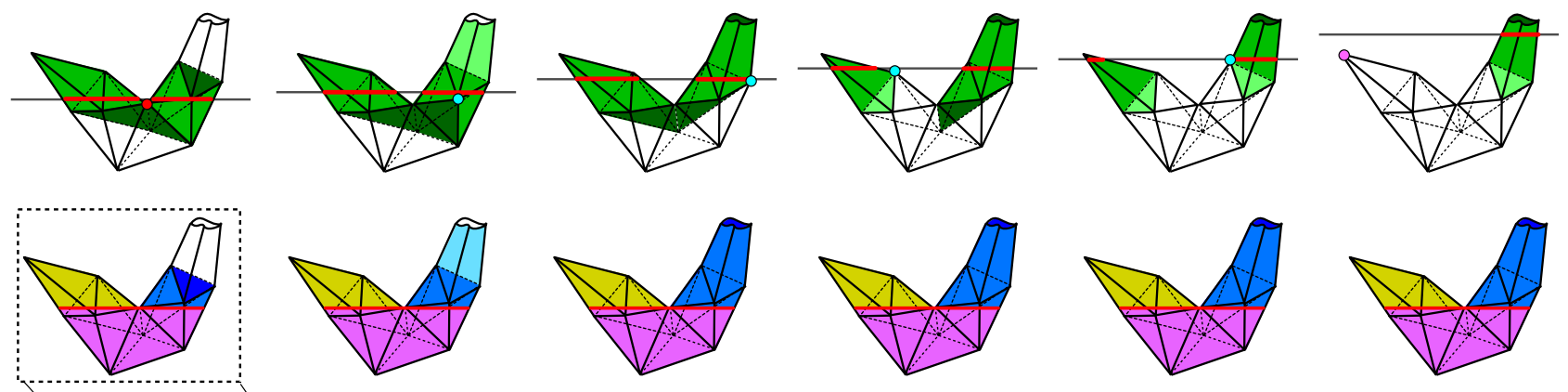

(h)

(j)

(k)

(I)

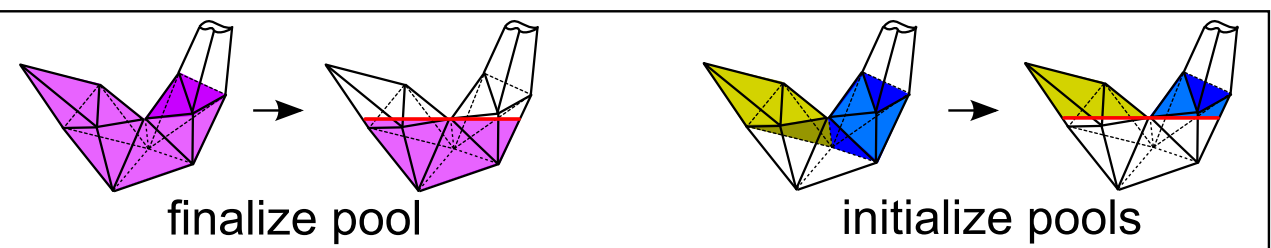

$\circ$ beginning vertex $\circ$ ending vertex $\circ$ no-change vertex $\bullet$ merge/split vertex

Fig. 4. Figures (a)-(l) show how pools are constructed according to generation, completion, and updating of boundary cycles. The top drawing in each subfigure shows the set of triangles in boundary cycles just after the sweep plane processes the indicated vertex as in Figure 2; the bottom drawing shows the corresponding construction of pools.
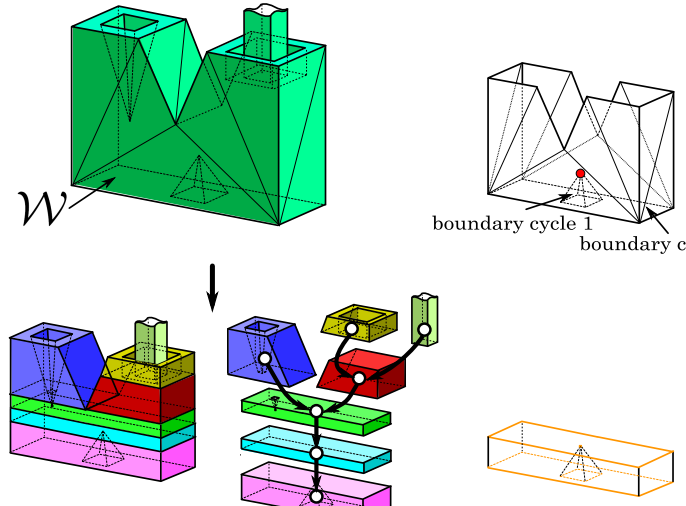

(a)
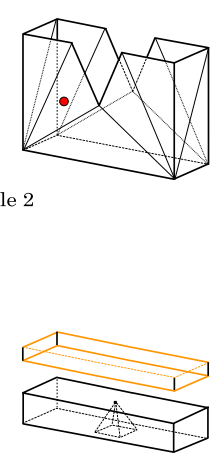

(b)
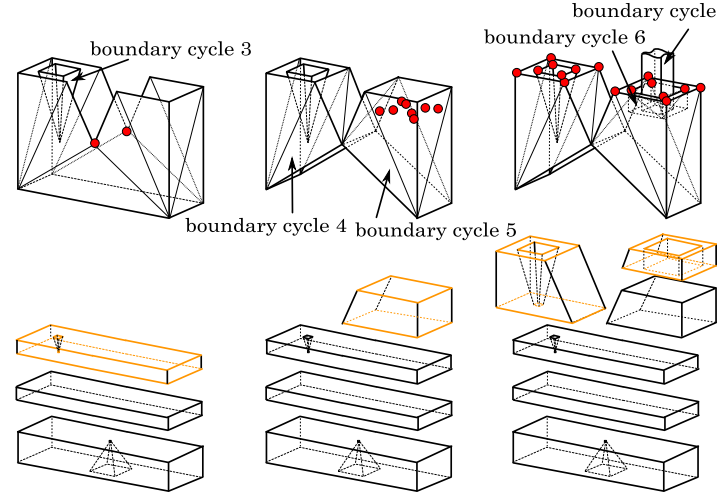

(e)

Fig. 5. Figures (a)-(e) show how pools are constructed according to generation, completion, and updating of boundary cycles in general pool segmentation. We segment $\mathcal{W}$ into pools where the topology of slice polygon changes. The top drawing in each subfigure shows the boundary cycles just before processing the indicated vertices; the bottom drawing shows the pools already completed just after processing the indicated vertices. The line segments shown in orange indicate the bottom face and top face of just completed pools. Notice that, for each pool, the bottom face and top face is defined by the triangles in the same set of boundary cycles.

pearance of the inner slice polygon boundary just changes the topology of the existing slice polygon. However, to simplify our implementation (as well as the volume compu- tation described below, we segment $\mathcal{W}$ into pools whenever a boundary cycle is generated or completed (which is equivalent to saying whenever the topology of a slice poly- 
gon changes). When a slice polygon appears, disappears, merges, or splits, the corresponding slice polygon boundary also appears, disappears, merges, or splits, and thus at least one boundary cycle is generated or completed. Therefore, the modified segmentation rule satisfies our original pool segmentation criteria.

Thus, a pool is initialized and finalized when an outer boundary cycle defining the pool is generated and completed in the same manner as in the simplified case. In addition, given a pool, every time one of the inner boundary cycles defining the pool is generated or completed, we finalize the pool and initialize a new one. Using this segmentation rule, each pool is entirely defined by the same set of boundary cycles, i.e. the set of boundary cycles when the pool is initialized and finalized is the same (although their triangles will have changed if any no-change vertices are on the boundary between the bottom and top face).

Subfigures 5 (a)-(e) show the boundary cycles just before processing the indicated vertices and the pools already completed just after processing the indicated vertices. In Figure 5 (a), boundary cycle 1, an inner boundary cycle, completes. We finalize the first pool defined by outer boundary cycle 2 immediately enclosing boundary cycle 1 . Since outer boundary cycle 2 is not completed, we initialize a new pool defined by outer boundary cycle 2 . In Figure 5 (b), boundary cycle 3, an inner boundary cycle, is generated. We associate boundary cycle 3 with boundary cycle 2 , its enclosing boundary cycle. Since boundary cycle 2 has already defined a pool not finalized yet, we finalize that pool, and initialize a new pool defined by boundary cycle 2 and boundary cycle 3 . In Figure 5 (c), boundary cycle 2 splits into boundary cycle 4 and boundary cycle 5 . We finalize the existing pool defined by boundary cycle 2 . Since inner boundary cycle 3 , immediately enclosed by boundary cycle 2 , is not completed, we reassociate boundary cycle 3 with boundary cycle 4 , which immediately encloses boundary cycle 3 just above the indicated vertices. We initialize a new pool defined by boundary cycle 4 and boundary cycle 3 (immediately enclosed by it), and boundary cycle 5 , respectively. In Figure 5 (d), boundary cycle 6 , an inner boundary cycle, and boundary cycle 7 , an outer boundary cycle, are generated. We associate boundary cycle 6 with boundary cycle 5, its enclosing boundary cycle. Since boundary cycle 5 has already defined a pool not finalized yet, we finalize that pool, and initialize a new pool defined by boundary cycle 5 and boundary cycle 6 . We also initialize a new pool defined by boundary cycle 7 . In Figure 5 (e), boundary cycle 4 and boundary cycle 3 (immediately enclosed by it), and boundary cycle 5 and boundary cycle 6 (immediately enclosed by it) are completed. We finalize the pool defined by these boundary cycles.

Now, we give the algorithm to implement this segmentation rule. For a given $z$ where $V(z) \neq \emptyset$, let $G_{\text {outer }}$ and $G_{\text {inner }}$ be the sets of new outer and inner boundary cycles, respectively, generated at $v \in V(z)$, and $C_{\text {outer }}$ and $C_{\text {inner }}$ be the sets of existing outer and inner boundary cycles, respectively, completed at $v \in V(z)$. Then, a pool is constructed by the following rules.

For a given $z$ where $V(z) \neq \emptyset$ :

(i) For each updated boundary cycle $b \in U$, we add new triangles inserted into $b$ at $z$ to the pool defined by $b$.

(ii) For each completed outer boundary cycle $b_{\text {outer }} \in$ $C_{\text {outer }}$, we finalize the pool defined by $b_{\text {outer }}$. We let inner $\left(b_{\text {outer }}\right)$ be the set of inner boundary cycles immediately enclosed by $b_{\text {outer }}$ at $z^{-}$. For each $b_{\text {inner }} \in$ inner $\left(b_{\text {outer }}\right)$, if $b_{\text {inner }} \notin C_{\text {inner }}$, we add $b_{\text {inner }}$ to $G_{\text {inner }}$ (Figure $5(\mathrm{c})(\mathrm{e})$ ).

(iii) For each completed inner boundary cycle $b_{\text {inner }} \in$ $C_{\text {inner }}$, we let $b_{\text {outer }}$ be the outer boundary cycle immediately enclosing $b_{\text {inner }}$ at $z^{-}$. We dissociate $b_{\text {inner }}$ from $b_{\text {outer. }}$. If the pool defined by $b_{\text {outer }}$ is not finalized, before the dissociation, we finalize the pool and add $b_{\text {outer }}$ to $G_{\text {outer }}$ (Figure $5(\mathrm{a})$ ).

(iv) For each newly generated inner boundary cycle $b_{\text {inner }} \in G_{\text {inner }}$, we find the outer boundary cycle $b_{\text {outer }}$ immediately enclosing $b_{\text {inner }}$ at $z^{+}$. We associate $b_{\text {inner }}$ with $b_{\text {outer }}$. If the pool defined by $b_{\text {outer }}$ is not finalized, before the association, we finalize the pool and add $b_{\text {outer }}$ to $G_{\text {outer }}$ (Figure $5(\mathrm{~b})(\mathrm{d})$ ).

(v) For each newly generated outer boundary cycle $b_{\text {outer }} \in G_{\text {outer }}$, we initialize a new pool $p$ defined by $b_{\text {outer }}$ and its inner boundary cycles immediately enclosed by $b_{\text {outer }}$ at $z^{+}$.

(vi) Let $P_{\text {init }}$ and $P_{\text {final }}$ be the sets of pools initialized and finalized at $z$, respectively. If $P_{\text {init }} \neq \emptyset$ and $P_{\text {final }} \neq$ $\emptyset$, for $p_{i} \in P_{\text {init }}$ and for $p_{f} \in P_{\text {final }}$, we compare the bottom face of $p_{i}$ and the top face of $p_{f}$. If they overlap, we add a directed edge from the node corresponding to $p_{i}$ to the node corresponding to $p_{f}$ in the directed graph.

Algorithm 3 shows the corresponding pseudocode. InitializePool and FinalizePool were shown in Algorithm 1 and 2, respectively. Process Vertices takes $V(z)$ as input and returns a set of boundary cycles generated, completed, and updated at $z$, respectively. ConstructConnectivity compares the bottom face(s) of generated pool(s) and the top face(s) of completed pool(s) by performing a 2D polygon intersection test. ClassifyBoundaryCycle classifies each newly generated boundary cycle as either an outer boundary cycle or an inner boundary cycle using ray shooting to implement a winding number test [5].

\section{PREDICTING WATER TRAP REGIONS}

After completing the sweep from $\mathrm{z}=-\infty$ to $\mathrm{z}=+\infty$, the space $\mathcal{W}$ is segmented into pools that are connected to each other if their bottom faces and top faces are overlapping. As we described above, given a pool, if there is no path from the corresponding node to the node corresponding to the bottommost pool, the pool is a potential water trap region (depending on inflow location). The bottommost pool corresponds to the first pool created during sweeping.

Finding the pools that are potential water trap regions is 


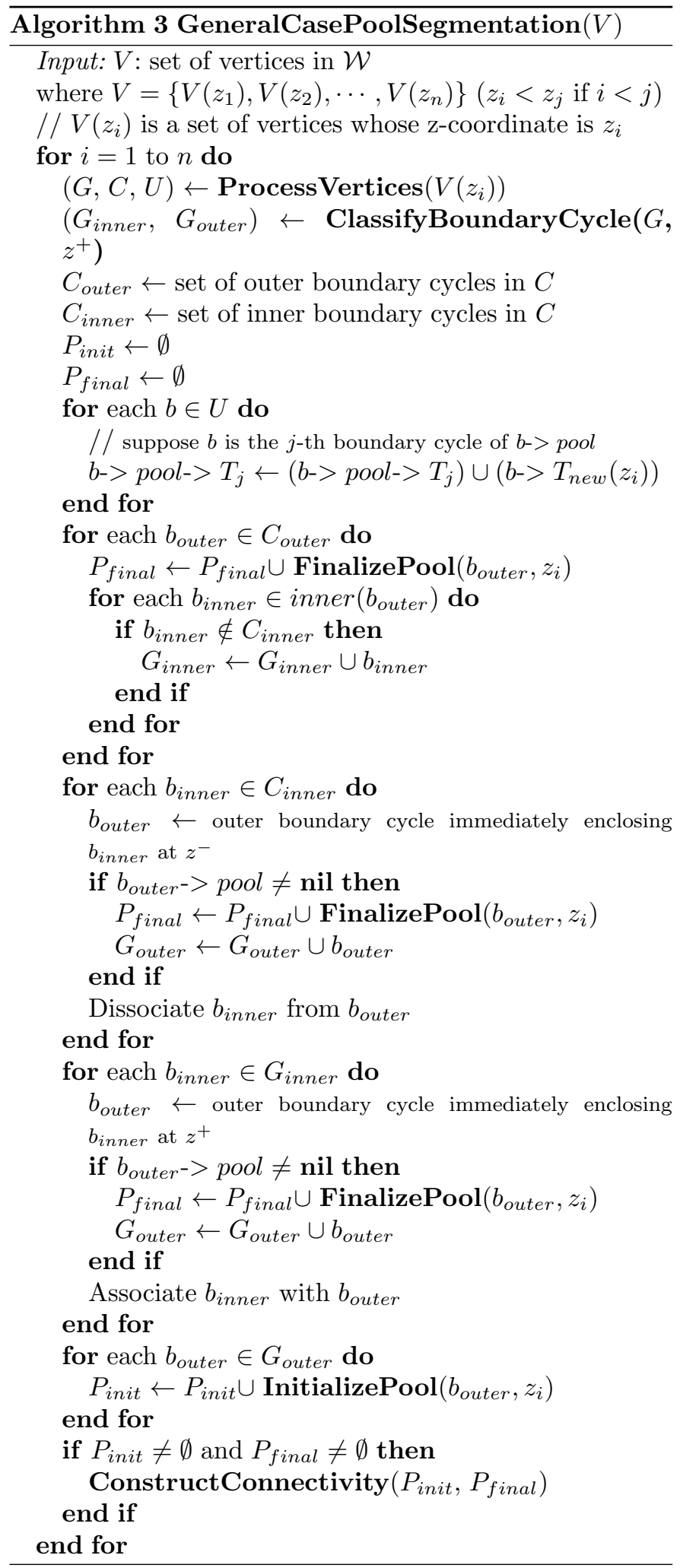

straightforward. From the node corresponding to the bottommost, we traverse the graph in the opposite direction of the graph edges until we have visited all reachable nodes. The nodes we cannot reach from the node corresponding to the bottommost pool represent potential water trap regions. For the traversal from the bottommost node, we do not have to visit the same node twice; therefore, the time complexity of the procedure is linear with respect to the number of pools.

\section{Quantitative Evaluation of a Part Orientation}

Since we can compute the volume of water each pool can hold, we can also quantitatively evaluate a given part orientation by summing the volumes of pools that are determined to be water trap regions.

The volume of an arbitrary polyhedron defined by a set of triangles $T$ can be computed using equation (1), where each triangle $t \in T$ is defined by the points $v_{t 1}, v_{t 2}, v_{t 3}$ ordered counterclockwise when viewed from the exterior of the polyhedron.

$$
V=\frac{1}{6} \sum_{t \in T}\left(v_{t 1} \times v_{t 2} \cdot v_{t 3}\right)
$$

Our pools are bounded on the side by original and trimmed triangles from $\mathcal{W}$ and on the bottom and top by 2D polygons, possibly with holes. Given a pool defined by $n$ boundary cycles, we let the vertices constituting the $i$-th closed polygonal chain of the bottom face be $l_{i j}(1 \leq j \leq$ $\left.p_{i}\right)$, and the vertices constituting the $i$-th closed polygonal chain of the top face be $u_{i j}\left(1 \leq j \leq q_{i}\right)$, with the vertices of the outer and inner slice polygon boundaries enumerated in counter-clockwise and clockwise order, respectively, when viewed from the exterior of the pool.

Then the volume of this pool can be computed using equation (2):

$$
\begin{aligned}
V_{\text {pool }} & =\frac{1}{6}\left\{\sum_{t \in \text { pool }}\left(v_{t 1} \times v_{t 2} \cdot v_{t 3}\right)+\sum_{i=1}^{n} \sum_{j=2}^{p_{i}-1}\left(l_{i 1} \times l_{i j} \cdot l_{i j+1}\right)\right. \\
& \left.+\sum_{i=1}^{n} \sum_{j=2}^{q_{i}-1}\left(u_{i 1} \times u_{i j} \cdot u_{i j+1}\right)\right\}
\end{aligned}
$$

\section{RESULTS AND DISCUSSION}

Figures $6-8$ shows the result of our segmentation and the identified water trap regions using the presented method on industrial cylinder head models. Table 1 shows timing data for pool segmentation and directed graph construction on the parts illustrated. The timing was performed on a computer with a $2.66 \mathrm{GHz}$ Intel Core i7 CPU with $4 \mathrm{~GB}$ of memory. Running times increase with the number of vertices but not necessarily with the number of generated pools, since the algorithm is also sensitive to the complexity of the geometry of the pools. Timing is shown for each part in two extreme orientations. We have found that the same part in a different orientation can have twice as many pools, and three times the running time for pool segmentation and graph construction. Once we obtain segmented pools and the corresponding directed graph, our algorithm to identify water trap regions for a given inflow location takes less than a millisecond, fast enough for even the most complex models. The directed graph our algorithm constructs has by nature more information than the corresponding Reeb graph of a 3-manifold with boundary with respect to the 
Table 1

Timing for pool segmentation and graph construction.

\begin{tabular}{|r|r|r|r|r|}
\hline & $\begin{array}{r}\text { cylinder_- } \\
\text { head1, 1st } \\
\text { orientation }\end{array}$ & $\begin{array}{r}\text { cylinder_- } \\
\text { head1, 2nd } \\
\text { orientation }\end{array}$ & $\begin{array}{r}\text { cylinder_ } \\
\text { head2, 1st } \\
\text { orientation }\end{array}$ & $\begin{array}{r}\text { cylinder } \\
\text { head2, 2nd } \\
\text { orientation }\end{array}$ \\
\hline \# vertices & 104,310 & 104,310 & 144,546 & 144,546 \\
\hline \# pools & 824 & 706 & 876 & 1552 \\
\hline time (sec.) & 0.827 & 2.661 & 2.005 & 3.855 \\
\hline
\end{tabular}

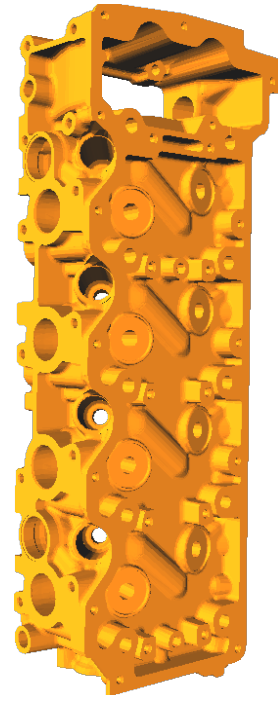

(a)

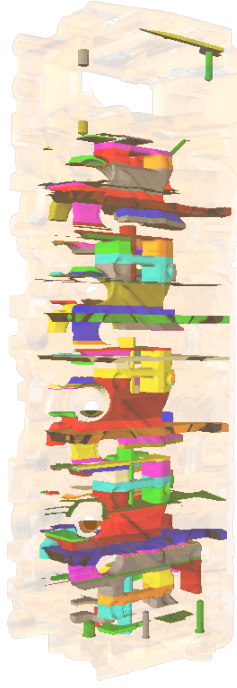

(b)
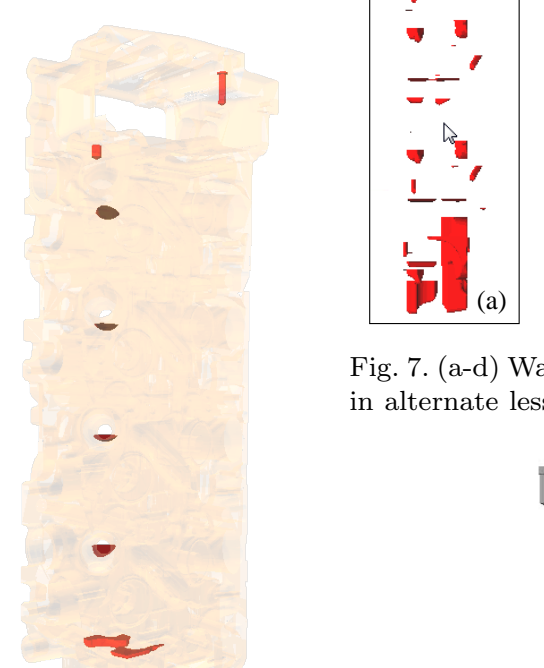

(c)

Fig. 6. We applied our algorithm to the cylinderhead_1 workpiece shown in (a). (b) Pool segmentation of the workpiece. Pools are assigned random colors. (c) Water trap regions of the workpiece.

height function. While our segmentation rule takes into account all the topology changes of $2 \mathrm{D}$ slices, the Reeb graph does not capture them; the Reeb graph only captures the merging and splitting of connected components. Given a geometry and our directed graph, we can easily obtain the corresponding Reeb graph by deleting nodes that have only one node connected above and one node connected below, respectively.

\section{CONCLUSION}

In this paper, we proposed a new pool segmentation data structure and algorithm based on topological changes of $2 \mathrm{D}$ slices with respect to gravity direction. We showed that we can predict potential water trap regions of a given geometry by analyzing the directed graph based on the segmented pools. In the future, we plan to utilize this data structure to accelerate physics-based simulation of fluid flow inside mechanical parts with complex geometry. Although recent advances in CPUs and GPUs make real-time fluid flow simulation possible in a simple computational domain, performing such simulation in a complex domain in real-time is still challenging. We believe that our pool segmentation data structure may also prove useful for other applications analyzing fluid flow inside complex geometry.
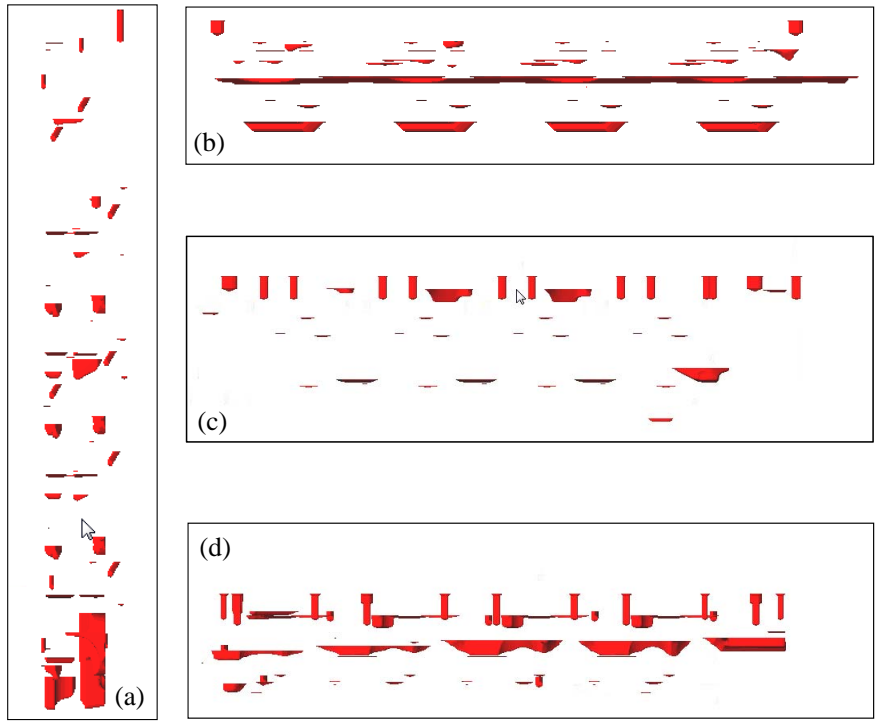

(d)

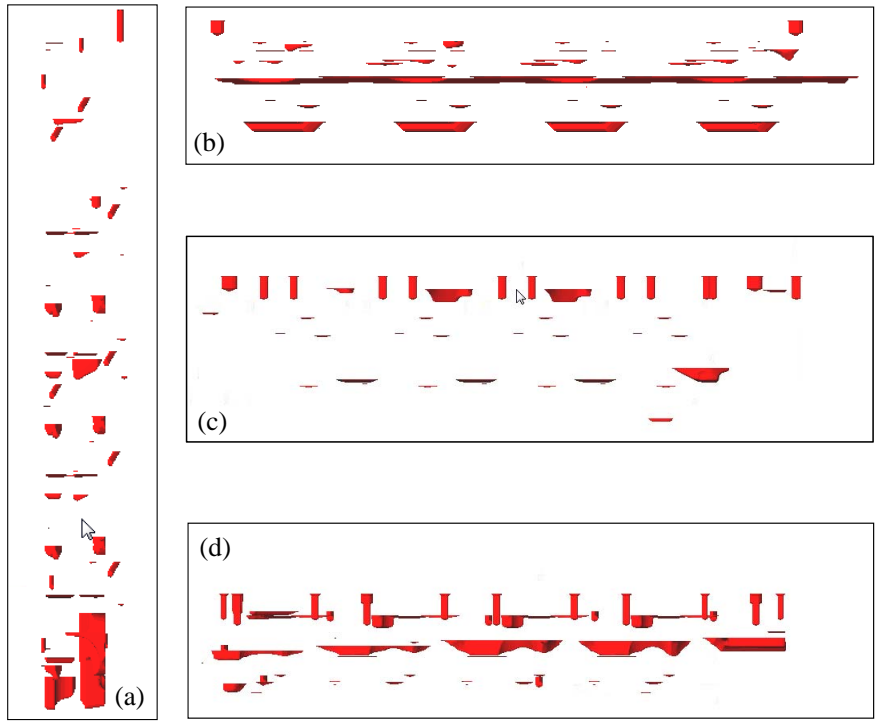

Fig. 7. (a-d) Water trap regions of the same cylinderhead_1 workpiece

in alternate less desirable orientations.

\section{II}

(a)
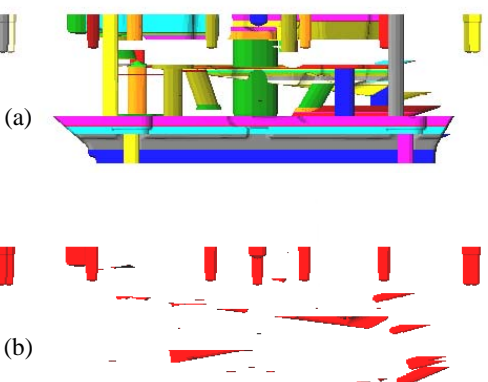

Fig. 8. (a) Pool segmentation of the cylinderhead_2 workpiece. (b) Water trap regions of the workpiece.

\section{ACKNOWLEDGMENTS}

Cylinder head models appear courtesy of Daimler. We also would like thank Klaus A. Berger, Adarsh Krishnamurthy, Wei Li, and Sushrut Pavanaskar for valuable feedback. This material is based on work supported in part by Daimler, UC Discovery under Grant No. DIG07-10224, and the National Science Foundation under Grant No. 0621198.

\section{References}

[1] G. Aloupis, J. Cardinal, S. Collette, F. Hurtado, S. Langerman, and J. O'Rourke. Draining a polygonorrolling a ball out of a polygon. Computational geometry, 47(2):316-328, 2014.

[2] D. Arbelaez, M. Avila, A. Krishnamurthy, W. Li, Y. Yasui, D. Dornfeld, and S. McMains. Cleanability of mechanical components. In Proceedings of 2008 NSF Engineering Research and Innovation Conference, 2008.

[3] M. Avila, C. Reich-Weiser, D. Dornfeld, and S. McMains. Design and manufacturing for cleanability in high performance cutting. In Proceeding of 2nd International High Performance Cutting Conference, 2006.

[4] P. Bose and G. Toussaint. Geometric and computational aspects of gravity casting. Computer-aided Design, 27(6):455-464, 1995.

[5] M. de Berg, M. van Kreveld, M. Overmars, and O. Schwarzkopf. Computational Geometry: Algorithms and Applications. Springer-Verlag, third edition, 2008. 


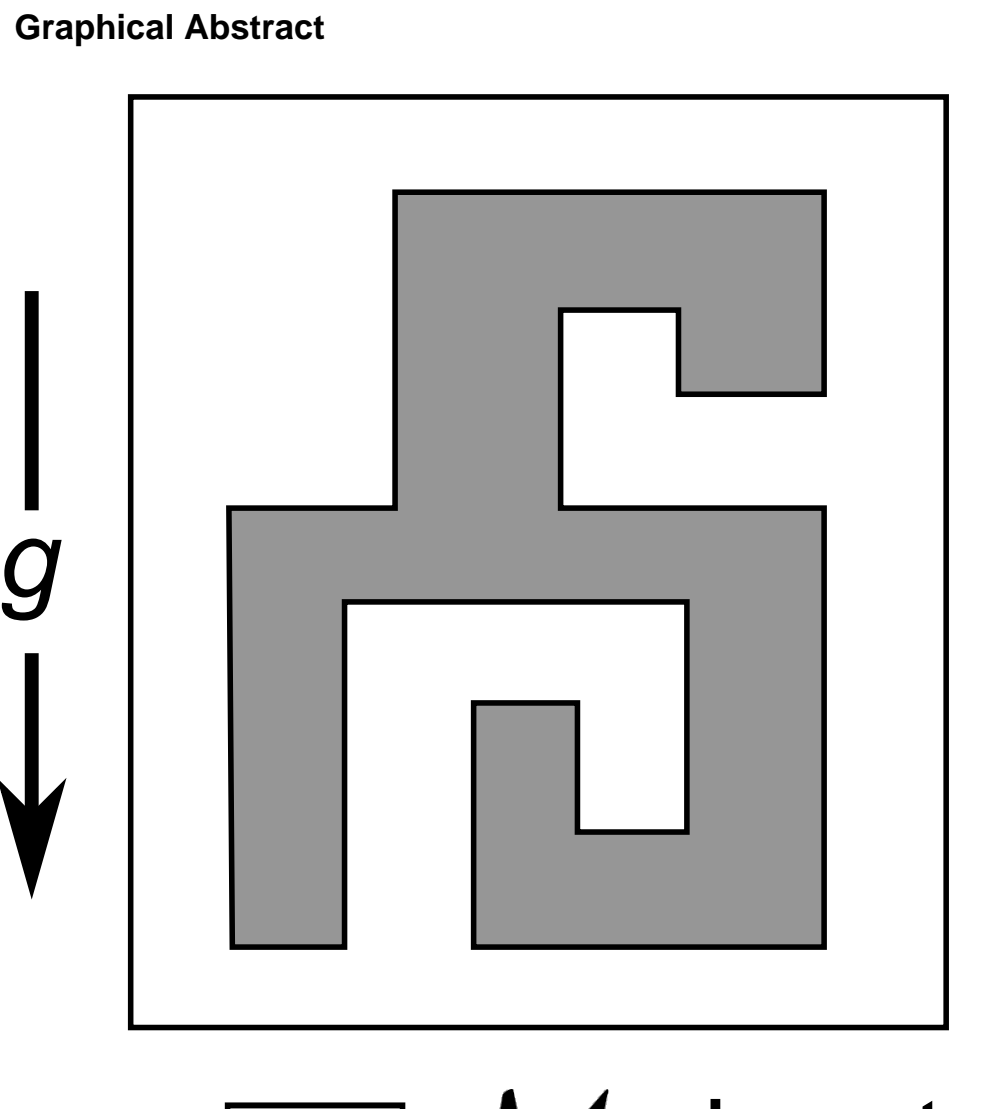

$\square \mathcal{M}$ : input

(a)

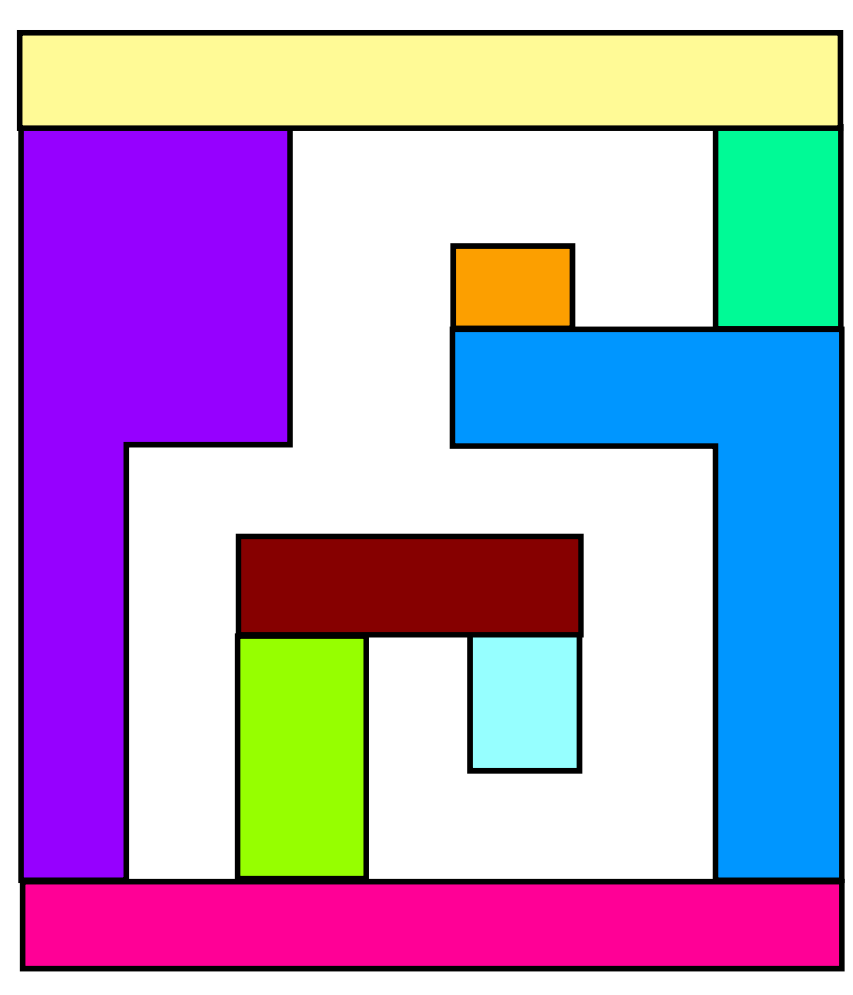

(e)

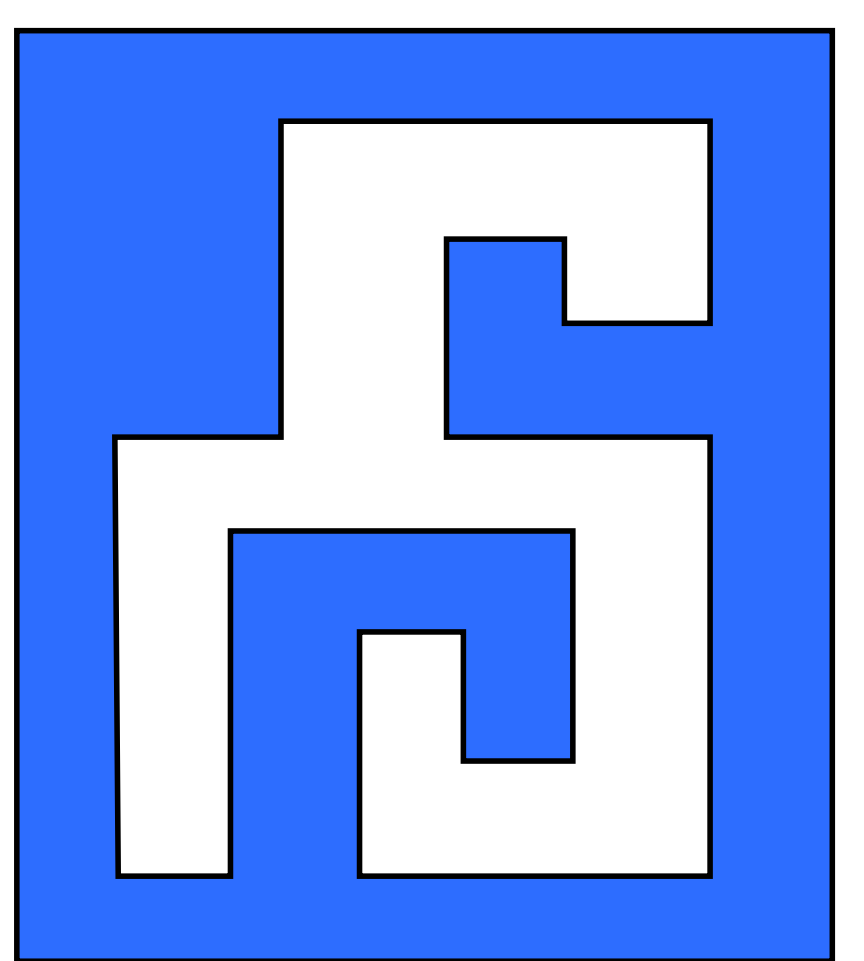

$\square \mathcal{W}$

(b)

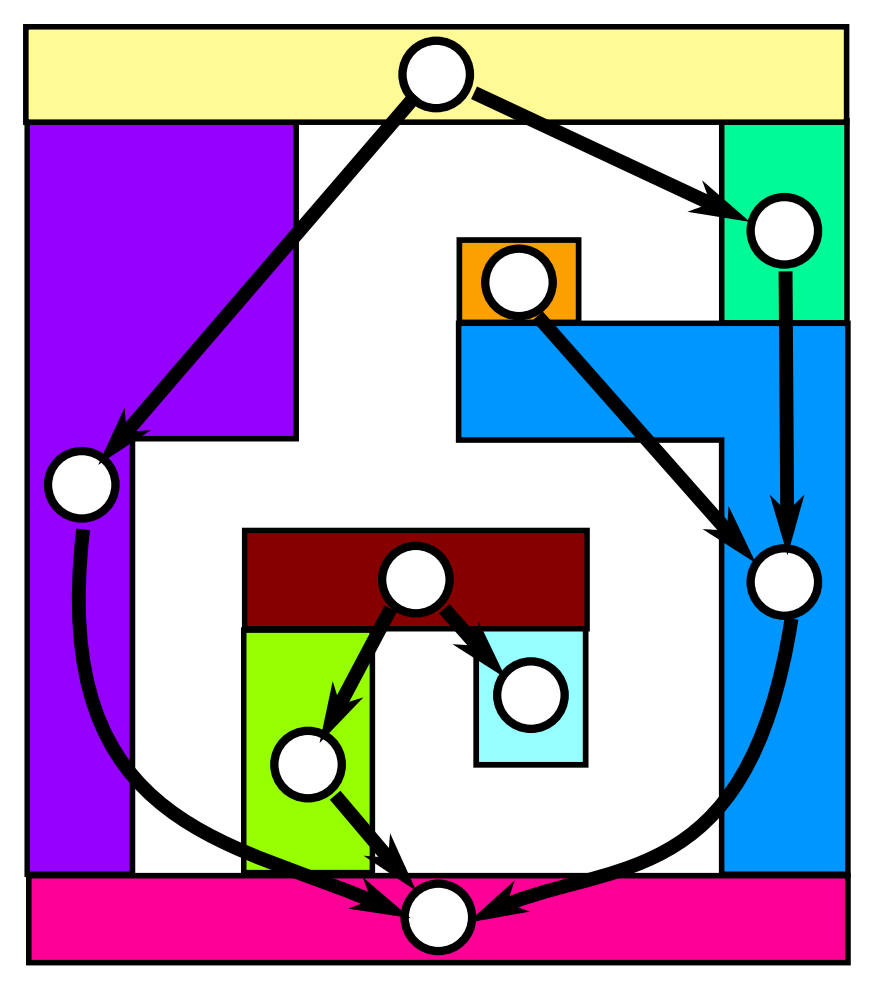

(f)

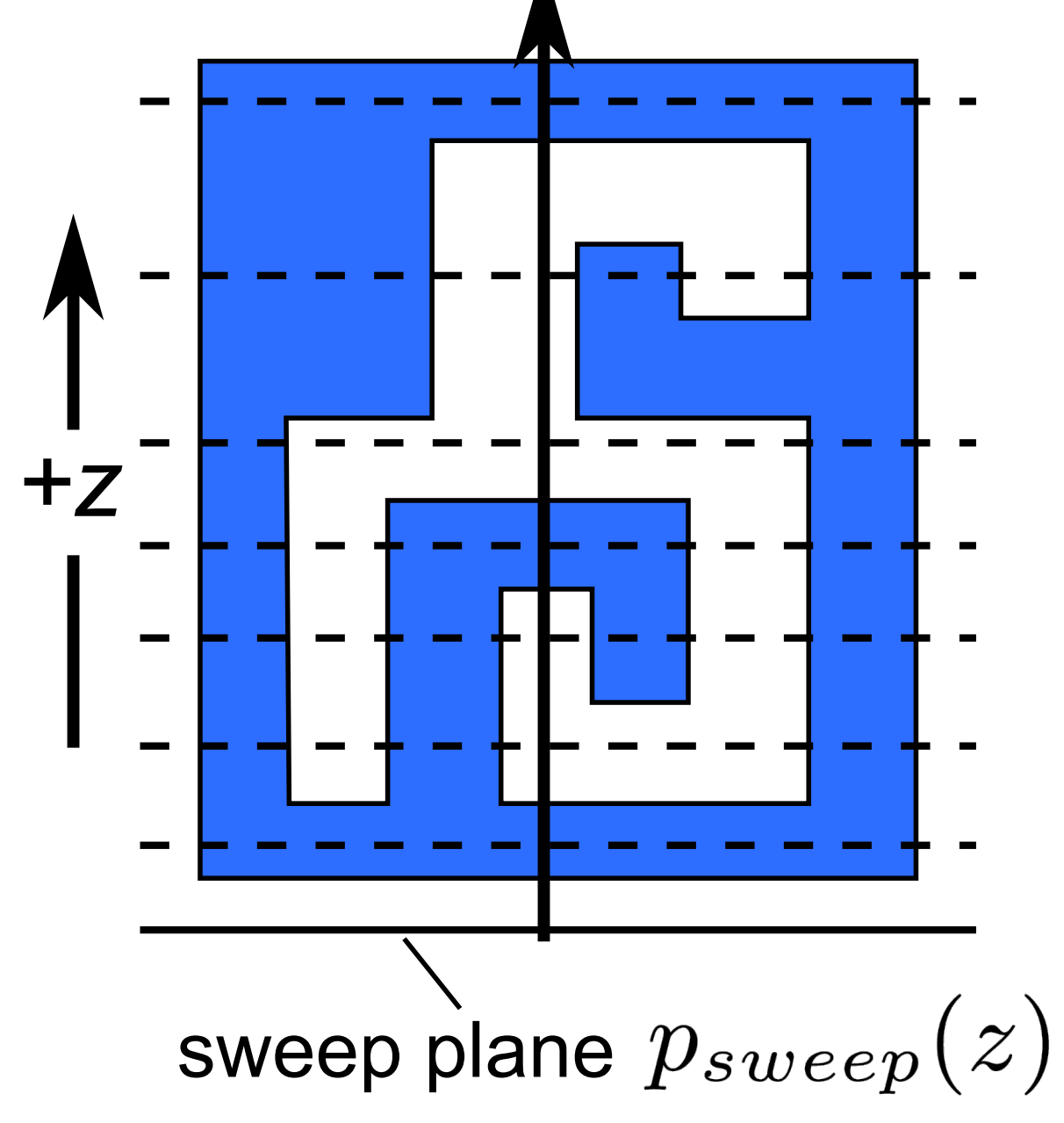

(c)

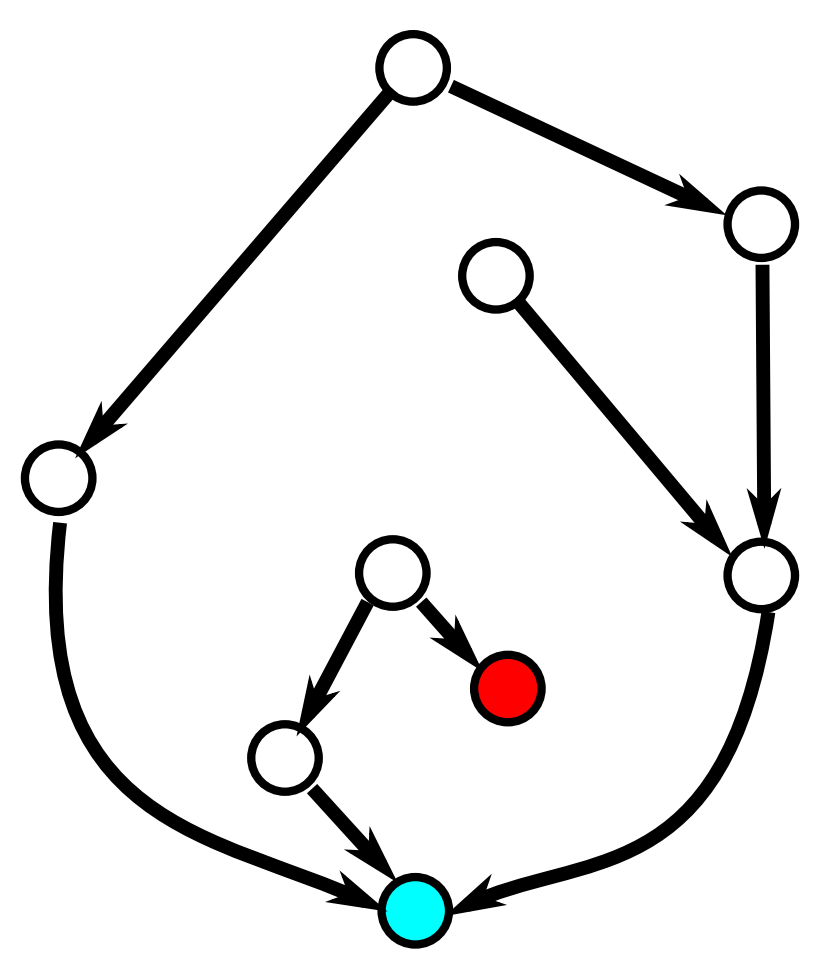

(g)

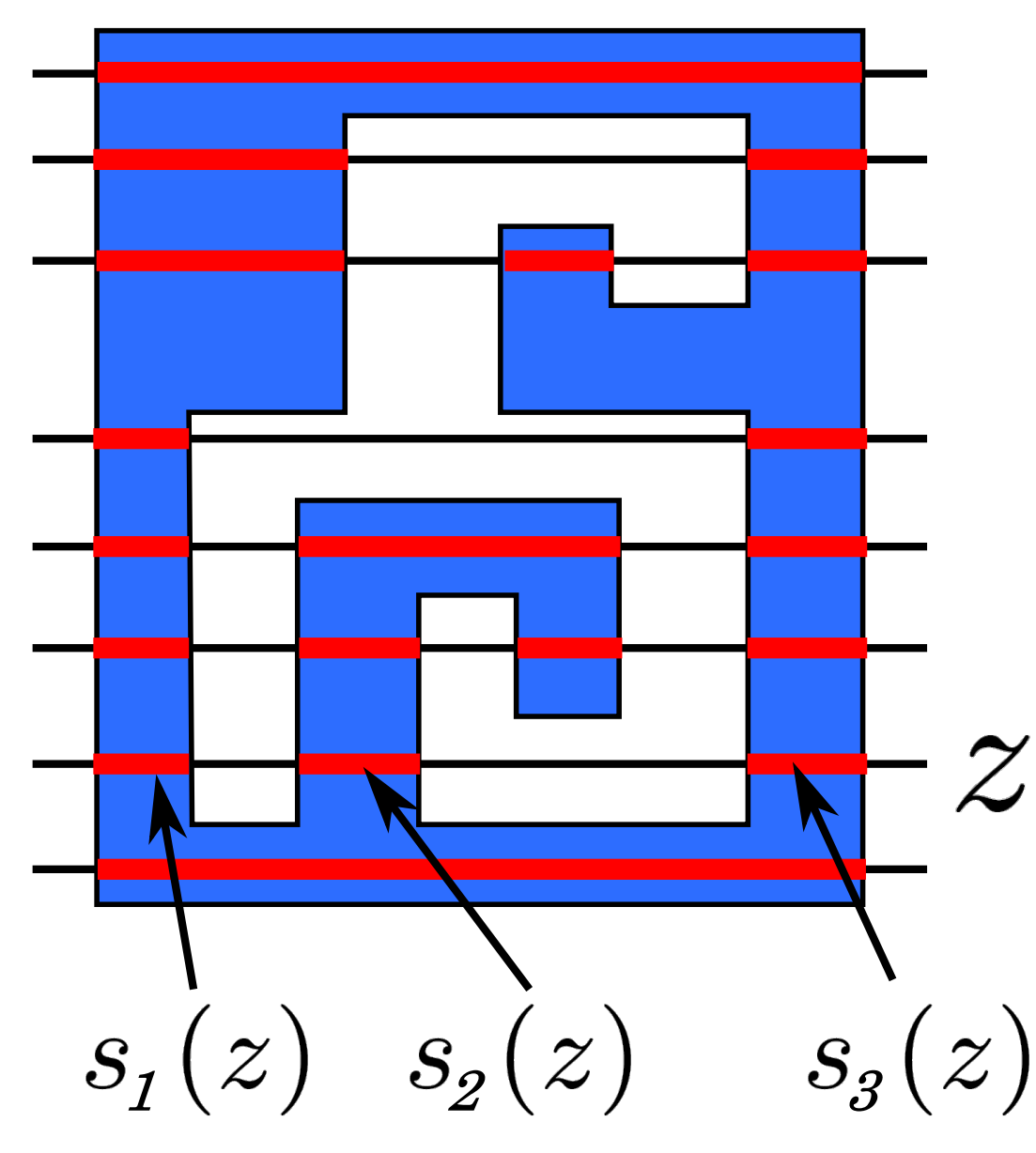

(d)

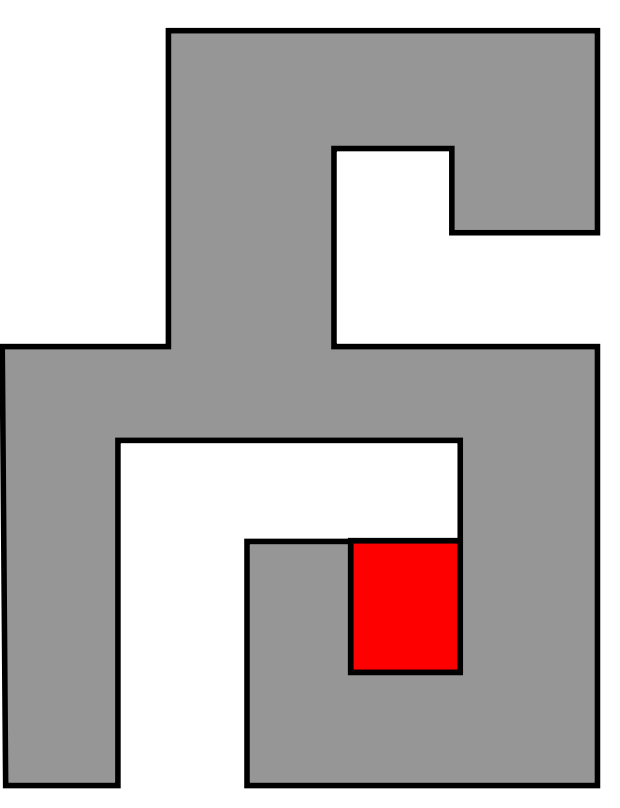

(h) 